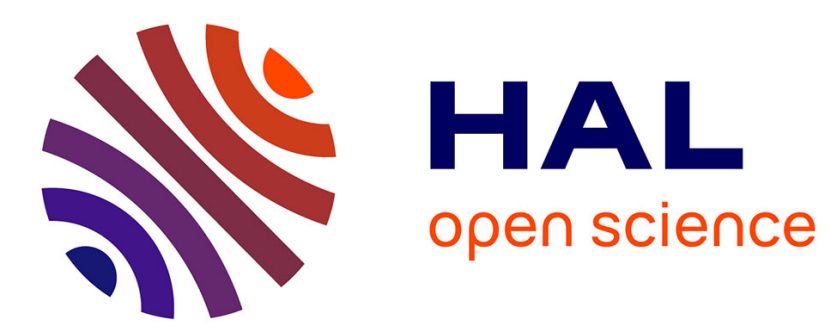

\title{
Evidence for a reversible microcrystal-quasicrystal phase transition in decagonal Al-Cu-Co(-Si)
}

\author{
M. Fettweis, Pascale Launois, R. Reich, R. Wittmann, F. Denoyer
}

\section{To cite this version:}

M. Fettweis, Pascale Launois, R. Reich, R. Wittmann, F. Denoyer. Evidence for a reversible microcrystal-quasicrystal phase transition in decagonal Al-Cu-Co(-Si). Phys.Rev.B, 1995, B 51. hal03330046

\section{HAL Id: hal-03330046 \\ https://hal.science/hal-03330046}

Submitted on 31 Aug 2021

HAL is a multi-disciplinary open access archive for the deposit and dissemination of scientific research documents, whether they are published or not. The documents may come from teaching and research institutions in France or abroad, or from public or private research centers.
L'archive ouverte pluridisciplinaire HAL, est destinée au dépôt et à la diffusion de documents scientifiques de niveau recherche, publiés ou non, émanant des établissements d'enseignement et de recherche français ou étrangers, des laboratoires publics ou privés. 
Phys. Rev. B 51, 6700 (1995)

\title{
Evidence of a reversible microcrystal-quasicrystal phase transition in decagonal $\mathrm{Al}-\mathrm{Cu}-\mathrm{Co}(-\mathrm{Si})$
}

\author{
M. Fettweis, P. Launois, R. Reich, R. Wittmann, and F. Dénoyer \\ Laboratoire de Physique des Solides, Université Paris-Sud, Bâtiment 510, 91405 Orsay Cédex, France
}

(Received 23 November 1994)

\begin{abstract}
Experimental evidence of a "first-order" reversible microcrystal-quasicrystal phase transition in decagonal $\mathrm{Al}_{63} \mathrm{Cu}_{17.5} \mathrm{Co}_{17.5} \mathrm{Si}_{2}$ is presented. High-resolution single-crystal $\mathrm{x}$-ray diffraction studies clearly show that (i) the low-temperature phase is microcrystalline and it is formed of coherent twins of the $(5,7)$ crystalline approximant with cell parameters $\left(a=b \approx 51.5 \AA, c \approx 4.13 \AA\right.$, and $\left.\gamma=108^{\circ}\right)$, and (ii) the high-temperature phase is the true quasicrystalline phase. Our results are in favor of a random-tiling description of the quasicrystalline state, i.e., for a phase stabilized by entropy effects.
\end{abstract}

The study of the thermal stability of quasicrystals is fundamental for the understanding of their growth process, the nature of their quasicrystalline order, and their physical properties. Although two-dimensional quasicrystals with decagonal symmetry, in particular decagonal $\mathrm{Al}-\mathrm{Cu}-\mathrm{Co}(-\mathrm{Si})$, have been the subject of many studies since their discovery, the state of knowledge concerning their stability is rather confused. Indeed, for the same nominal composition $\mathrm{Al}_{65} \mathrm{Cu}_{20} \mathrm{Co}_{15}$, some authors observed a quasicrystalline state ${ }^{1,2}$ and others a microcrystalline one. ${ }^{3,4}$ For a slightly different nominal composition $\mathrm{Al}_{63} \mathrm{Cu}_{17.5} \mathrm{Co}_{17.5} \mathrm{Si}_{2}$, a detailed analysis of "highresolution" $\mathrm{x}$-ray-diffraction patterns led us to conclude that our samples were in the same microcrystalline state: ${ }^{5}$ this state consists of coherent twinned domains (five variants) of the $(5,7)$ high-order approximant of the quasicrystal. Why the above-mentioned disagreements? Are they due to the method of preparation of the sample (nonequilibrium states, imperfectly reproducible steps of solidification, etc.) and/or are they due to the inability of some characterization methods to distinguish between microcrystalline and quasicrystalline states? The problem concerning the stability (or metastability) of the quasicrystalline state has not been cleared up by experiments carried out as a function of temperature. Based on in situ sample heating powder neutron diffraction ${ }^{6}$ or high-resolution electron microscopy (HREM) and electron diffraction, ${ }^{7}$ some authors claimed to have observed a phase transformation from a microcrystalline state to a quasicrystalline one. In Ref. 6, sharpening of peak widths above $700^{\circ} \mathrm{C}$ is said to "reflect a certain kind of ordering" in the microcrystalline structure, which does not prove that this "ordered state" is the quasicrystal. The electron microscopy experiments where the authors ${ }^{7}$ suppose to have observed a microcrystal to quasicrystal transformation above $900^{\circ} \mathrm{C}$ cannot be considered conclusive. Indeed, it is well known that in such a temperature range the vapor pressure of aluminum is high enough that experiments under vacuum are biased. Moreover, the electron microscopy experiments performed on samples annealed for a long time above $800^{\circ} \mathrm{C},{ }^{3}$ where the microcrystalline state is transformed into a quasicrystalline one, leave unanswered the questions concerning the microcrystal thermodynamical equilibrium and the stability temperature range of the quasi- crystal.

In order to settle this problem, we have undertaken single-crystal $\mathrm{x}$-ray-diffraction experiments as a function of temperature on a decaprismatic needle of nominal composition $\mathrm{Al}_{63} \mathrm{Cu}_{17.5} \mathrm{Co}_{17.5} \mathrm{Si}_{2}$. A reversible transformation between a low-temperature microcrystalline phase and a high-temperature quasicrystalline one is clearly observed in a decagonal system.

Before giving and discussing the experimental results, we recall that the sample studied is initially in the microcrystalline state at room temperature. ${ }^{5}$ Let us summarize what the microcrystalline state consists of, what the specificities of its diffraction patterns are, and what is expected in diffraction patterns if the microcrystal transforms into a quasicrystal.

We define a microcrystal as a state formed of small domains of an approximant crystalline phase of the quasicrystal, the domains being twinned in such a way that their orientational relationships build up perfectly the symmetry of the quasicrystal in reciprocal space. Note also that domains are thought to be coherently set, so that coherence lengths deduced from diffraction patterns can be greater than domain sizes. In the case of $\mathrm{Al}_{63} \mathrm{Cu}_{17.5} \mathrm{Co}_{17.5} \mathrm{Si}_{2},{ }^{5}$ the approximant crystalline state is the $(5,7)$ approximant, base-centered orthorhombic (rhombic unit-cell parameters at $T=300^{\circ} \mathrm{C}: a=b \approx 51.5$ $\AA, c \approx 4.13 \AA$, and $\gamma=108^{\circ}$ ); five variants exist, which are rotated relative to each other by $72^{\circ}$. The calculation of one $(5,7)$ crystalline approximant, ${ }^{5}$ derived from the structural quasicrystalline model of Steurer and $\mathrm{Kuo}^{2}$ and projected along its tenfold axis $(c)$, gives an image which can be compared to HREM images. ${ }^{8}$ Although the true unit cell is $a=b \approx 51.5 \AA$ and $\gamma=108^{\circ}$, an image issuing from a microdomain of one approximant can be locally ${ }^{8}$ interpreted as tiled with a cell of parameters $a=b \approx 51.5 \AA$ and $\gamma=144^{\circ}$, as found in Ref. 7. This remark may explain the discrepancy between Ref. 5 and Ref. 7.

The diffraction patterns are usually calculated by superimposing the five rotated reciprocal lattices corresponding to the five variants. ${ }^{3,5}$ The diffraction pattern has full decagonal symmetry in the $a^{*}, b^{*}$ plane. The very strong peaks of the approximant are so slightly shifted from the position of the corresponding peaks of the quasicrystal that the superimposition of the five variant 
diffraction patterns leads to sets of five very close peaks which, in the case of $\mathrm{Al}-\mathrm{Cu}-\mathrm{Co}(-\mathrm{Si})$ and within our experimental conditions, cannot be separated. ${ }^{5}$ Hence, for a transformation towards the quasicrystalline state, these very strong peaks will look unchanged. On the contrary, weaker diffraction peaks of the approximant are a little bit more shifted from the position for the quasicrystal and the resulting five peaks of the microcrystal can be separated. In this last case, for a transformation towards the quasicrystalline state, a single peak should appear inside this five-peak set.

The differences between quasicrystalline and microcrystalline diffraction patterns being very subtle, a good resolution combined with a high flux is necessary in order to unambiguously distinguish between the true quasicrystal and the microcrystal with large unit cell. ${ }^{5}$ For this reason, "single-crystal" $\mathrm{x}$-ray-diffraction experiments were performed at the synchrotron laboratory LURE (Orsay, France). A wavelength equal to $1.65 \AA$ was selected using the (111) reflection of a silicon monochromator. The precession technique was chosen because it supplies on a film the diffraction pattern of a given reciprocal plane without any deformation. Within our experimental conditions the resolution was about $800 \AA$. The sample was a decaprismatic needle extracted from an ingot of nominal composition $\mathrm{Al}_{63} \mathrm{Cu}_{17.5} \mathrm{Co}_{17.5} \mathrm{Si}_{2}$ (the ingot preparation method was published in Ref. 5). The sample was encapsulated in a silica capillary sealed under argon atmosphere, in order to prevent its oxidation and sublimation during the heating. High temperatures were obtained by blowing a stream of hot air on the capillary containing the decaprismatic needle, using a special device set up on the precession camera.

Three series of experiments have been performed. In the first, the decaprismatic needle was heated (heating rate: $35^{\circ} / \mathrm{min}$ ) and held at 700,750 , and $800^{\circ} \mathrm{C}$ for about $12 \mathrm{~h}$ at each temperature (film exposure time $\approx 10 \mathrm{~h}$ ). The upper limit has been chosen in order to avoid all problems of vaporisation and reaction with silica capillary. At the end of this experiment, the sample was quenched to room temperature by rapidly removing the capillary from the air flow. The $l=0$ decagonal diffraction patterns obtained at 700 and $800^{\circ} \mathrm{C}$ are shown in Fig. 1. Data fitting within the scope of the microcrystalline model and of the quasicrystalline model is reported in Fig. 2: at $700^{\circ} \mathrm{C}$, the sample is in the microcrystalline state $\left(a=b=52.0 \AA, \gamma=108^{\circ}\right)$ and at $800^{\circ} \mathrm{C}$, it has transformed into the quasicrystalline state. Peaks 1, 2, 3, and 4 in Fig. 1 are very strong peaks which look unchanged at 700,750 , and $800^{\circ} \mathrm{C}$. On the contrary, sets of peaks $A, B$, and $C$ change between 700 and $800^{\circ} \mathrm{C}$ as shown in Fig. 3. Each set of peaks is replaced by a single-quasicrystalline peak at $800^{\circ} \mathrm{C}$. At $750^{\circ} \mathrm{C}$, microcrystalline and quasicrystalline peaks are both observed, with no change in their respective positions compared to 700 and $800^{\circ} \mathrm{C}$ except for thermal effect: $:^{10}$ the quasicrystalline state coexists with the microcrystalline one, with perfect orientational relationships. The correlation lengths are found to be identical (resolution limited) at $700^{\circ} \mathrm{C}$ in the microcrystalline state, at $800^{\circ} \mathrm{C}$ in the quasicrystalline state, and at $750^{\circ} \mathrm{C}$, when the two states are

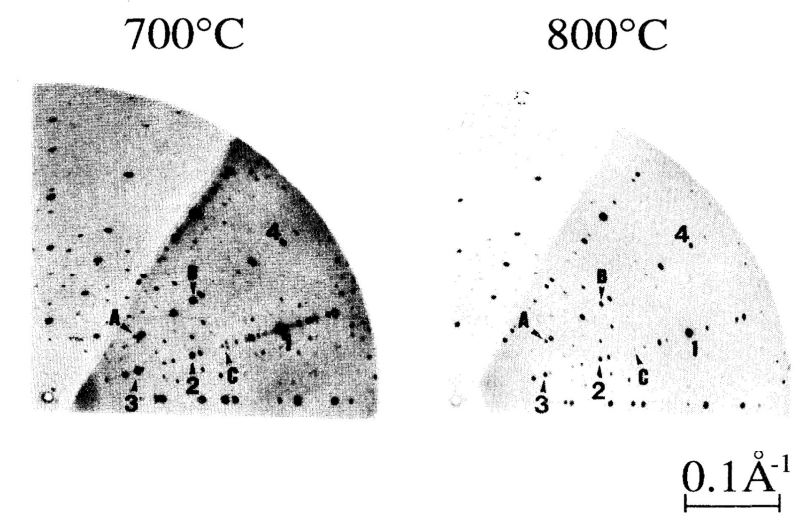

FIG. 1. Monochromatic x-ray precession patterns obtained from the $l=0$ reciprocal plane perpendicular to the periodic axis $c^{*}$ : at 700 and $800^{\circ} \mathrm{C}$ (only a part of the patterns is shown because of their tenfold symmetry). In order to distinguish between the diffraction peaks corresponding to $\lambda=1.65 \AA$ and those corresonding to $\lambda / n$ ( $n$ order reflections), a 1 -mm-thick aluminum sheet was placed in front of the film, giving rise to the clearest areas on the patterns. The aluminum absorption coefficients for $\lambda$ and $\lambda / n$ are such that only the diffraction peaks coming from $\lambda / n$ are visible on these clearest areas.

both present. Let us mention another experimental result, although it will not be discussed in this letter. In the $l=0$ plane, at $700^{\circ} \mathrm{C}$, diffuse scattering is clearly visible: for example, inside composed peaks such as sets $A$ and $B$, or as streaks radiating from the strong peak 1 . Such a phenomenon has almost completely disappeared at $750^{\circ} \mathrm{C}$.

Before the second experiment, we checked that the decaprismatic needle was still in the quasicrystalline state after the quenching. Then the decaprismatic needle was heated again (heating rate: $35^{\circ} / \mathrm{min}$ ) up to $700^{\circ} \mathrm{C}$ and held at this temperature for $19 \mathrm{~h}$, then cooled (cooling rate: $35^{\circ} / \mathrm{min}$ ) down to $650^{\circ} \mathrm{C}$ and held there for $17 \mathrm{~h}$. The two corresponding $l=0$ diffraction patterns are the same. It is concluded that relatively long annealings of the quasicrystalline state at these temperatures do not transform the quasicrystalline state into the microcrystalline one. At the end, the sample was heated up to $800^{\circ} \mathrm{C}$ and then quenched to room temperature.

Before starting the third experiment, the quasicrystalline state of the quenched sample was checked again. To test the reversibility of the microcrystal-quasicrystal transformation, the sample was heated $\left(35^{\circ} / \mathrm{min}\right)$ up to $800^{\circ} \mathrm{C}$, annealed at this temperature for $2 \mathrm{~h}$ and then slowly cooled down to room temperature (cooling rates: $0.19^{\circ} / \mathrm{min}$ between 800 and $295^{\circ} \mathrm{C}, 30^{\circ} / \mathrm{min}$ between $295^{\circ} \mathrm{C}$ and room temperature). An x-ray-diffraction pattern was then taken. All the peak positions of the microcrystalline state are rediscovered in this diffraction pattern [see Fig. 3(d)], obviously proving the reversibility of this solid transformation.

Thus, we have clearly observed a reversible phase transition between the microcrystalline state and the quasi- 

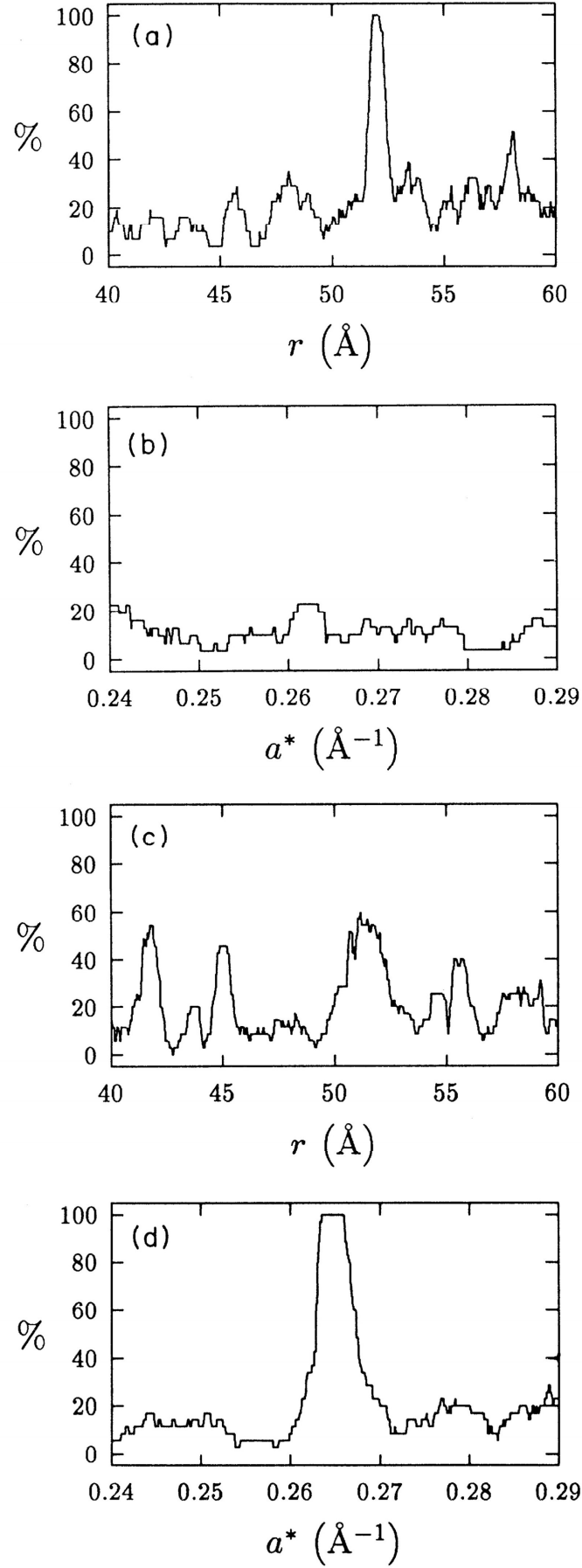

FIG. 2. Percentage of fitted peak positions (experimental accuracy: $0.002 \AA^{-1}$; reflections due to $\lambda / n$ have been suppressed) (A) for the initial state $\left(\mathrm{cf} .700^{\circ} \mathrm{C}\right.$ state), within the scope of (a) the microcrystalline model, (b) the quasicrystalline model, (B) for the transformed state (cf. $800^{\circ} \mathrm{C}$ state), within the scope of (c) the microcrystalline model, (d) the quasicrystalline model. The $x$ axis represents the $a=b$ parameter defined in the text for fitting with the microcrystalline model and the $a^{*}$ parameter defined in Ref. 2 for fitting with the quasicrystalline model, respectively. For the quasicrystalline model, indices of Ref. 2 vary between -9 and +9 .

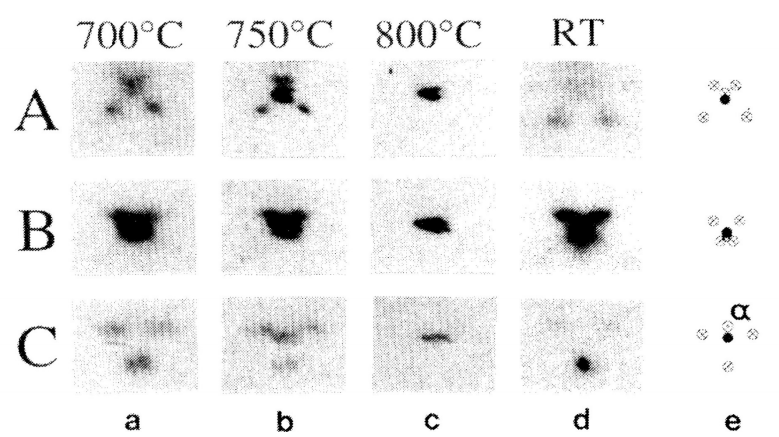

FIG. 3. Enlarged scale for peak sets $A, B$, and $C$ at (a) $700^{\circ} \mathrm{C}$; (b) $7500^{\circ} \mathrm{C}$; (c) $800^{\circ} \mathrm{C}$; and (d) room temperature (RT) after slow cooling from $800^{\circ} \mathrm{C}$; (e) corresponding calculated peak positions: crossed circles (within the scope of the microcrystalline model) and solid circles (within the scope of the quasicrystalline model). Measured peak positions of (a) and (d) are well fitted by the microcrystalline model. (Note that the calculated point $\alpha$ in set $C$ was not observed. It corresponds to an interference peak between two kinds of variants rotated relative to each other by $72^{\circ}$ : it may be extinct because of a destructive interference due to the coherence of domains.) Measured peak positions of (c) are fitted by the quasicrystalline model. The peaks in (b) comprise those in (a) plus those in (c).

crystalline one, the coexistence of the two states at $750^{\circ} \mathrm{C}$ (first experiment) and the hysteresis effect shown in the second experiment are features in favor of a first-order transition. The microcrystalline and the quasicrystalline states are found as the stable low-temperature and hightemperature phases, respectively. No transient state was observed.

Microcrystals, formed of twins of a high-order approximant phase, and quasicrystals present very similar atomic configurations and their strong diffraction peaks are nearly at the same positions in reciprocal space. From an energetical approach, using pair potential models or the Hume-Rothery criterion, one concludes that these two states are energetically very close. We have shown that they are both stable states, the microcrystal at low temperature and the quasicrystal at high temperature. This last result is in favor of a random tiling description of this quasicrystalline state, the quasicrystal then being entropically stabilized at high temperature. ${ }^{11}$

A natural question arises from our experiments: why is the $(5,7)$ base-centered orthorhombic approximant $\left(a=b \approx 52 \AA, \gamma=108^{\circ}\right)$ more stable than other highorder approximants, especially since the same order approximant is also observed in an Al-Co-Ni alloy? ${ }^{12}$

In conclusion, the existence of a reversible "first-order" orthorhombic microcrystal to quasicrystal transition is directly proved in decagonal $\mathrm{Al}-\mathrm{Cu}-\mathrm{Co}(-\mathrm{Si})$, the microcrystalline state being stable at low temperature and the quasicrystalline state at high temperature. Our results lead us to conjecture that the quasicrystalline phase, whether or not linked to the pseudocovalent character of this type of intermetallic alloy ${ }^{13}$ or to cluster packing, ${ }^{14}$ is a phase stabilized by entropic effects. 
The authors address their sincere acknowledgments to M. Lambert for her constant interest in this work and for helpful discussions. They are indebted to J. Doucet for the use of his D43 equipment of LURE. They are grateful to J. M. Godard for his efficient assistance in the ingot preparation and to D. Petermann for his peak position determination program. It is also a pleasure to thank B. Grushko and $\mathbf{M}$. Widom for interesting discussions and E. Cockayne for his critical reading of the manuscript. Laboratoire de Physique des Solides is "Unité associée au CNRS."
${ }^{1}$ L. X. He, Y. K. Wu, and K. H. Kuo, J. Mater. Sci. Lett. 7, 1284 (1988).

${ }^{2}$ W. Steurer and K. H. Kuo, Acta Crystallogr. B 46, 703 (1990).

${ }^{3}$ E. Ryba, S. Song, and L. Wang, J. Non-Cryst. Solids 153\&154, 623 (1993).

${ }^{4}$ M. Fettweis, P. Launois, F. Dénoyer, R. Reich, and M. Lambert (unpublished)

${ }^{5}$ M. Fettweis et al., Phys. Rev. B 49, 15573 (1994).

${ }^{6}$ C. Dong et al., J. Phys. Condens. Matter 3, 1665 (1991).

${ }^{7}$ M. Audier and R. Robertson, Philos. Mag. Lett. 64, 401 (1991).

${ }^{8}$ M. Fettweis, Ph.D. thesis, Université Paris XI-Orsay, France,
1994.

${ }^{9}$ R. Argoud and J. J. Capponi, J. Appl. Cryst. 17, 420 (1984).

${ }^{10}$ From the diffraction data, thermal expansion is found to be about $1.5 / 1000$ for a temperature increase of $100^{\circ} \mathrm{C}$.

${ }^{11}$ M. Widom, K. J. Strandburg, and R. H. Swenden, Phys. Rev. Lett. 58, 706 (1987); C. L. Henley, in Quasicrystals: The State of the Art, edited by D. P. DiVincenzo and P. Steinhardt (World Scientific, Singapore, 1991), p. 429.

${ }^{12}$ M. Kalning et al., J. Phys. Condens. Matter 6, 6177 (1994).

${ }^{13}$ R. Hu et al., Phys. Rev. B 46, 6105 (1992).

${ }^{14}$ C. Janot and M. de Boissieu, Phys. Rev. Lett. 72, 1674 (1994), 